\title{
Performance of pneumococcal urinary antigen test in patients with community-onset pneumonia: a propensity score-matching study
}

Jonghoo Lee ${ }^{1, \star}$ and Jae-Uk Song ${ }^{2, *}$

${ }^{1}$ Department of Internal Medicine, Jeju National University Hospital, Jeju; ${ }^{2}$ Division of Pulmonary and Critical Care Medicine, Department of Internal Medicine, Kangbuk Samsung Hospital, Sungkyunkwan University School of Medicine, Seoul, Korea

Received: December 22, 2018

Revised : April 3, 2019

Accepted: May 8, 2019

\section{Correspondence to}

Jonghoo Lee, M.D.

Department of Internal

Medicine, Jeju National

University Hospital, 15 Aran

13-gil, Jeju 63241, Korea

Tel: +82-64-717-1601

Fax: +82-64-717-1131

E-mail: lovlet@jejunu.ac.kr

*These authors contributed equally to this work.
Background/Aims: Although pneumococcal urinary antigen tests (PUATs) have universally been used for the diagnosis of pneumococcal pneumonia, data on the efficacy of these exams are limited. The objective of our study was to investigate the clinical impact of the PUAT in patients with community-onset pneumonia (CO-pneumonia).

Methods: We conducted a retrospective cohort study of patients diagnosed with CO-pneumonia. Patients were classified according to their PUAT results and were matched using the propensity score-matching method. The primary outcome was 30-day mortality.

Results: A total of 1,257 patients were identified and 163 (13.0\%) demonstrated positive PUAT results. The sensitivity and specificity values of PUAT for overall pneumococcal pneumonia were $56.5 \%$ and $91.4 \%$, respectively. In the full cohort, there were no significant differences in 30-day mortality between the two groups (6.1\% in the positive PUAT group vs. $8.2 \%$ in the negative PUAT group, $p=0.357$ ). However, in the propensity-matched cohort, the 30 -day mortality rates were lower in the positive PUAT group (5.6\% vs. $17.4 \%, p=0.001$ ). With respect to secondary outcomes, the proportion of patients with potentially drug-resistant pathogens, changes in antibiotics, and failure rates of initial antibiotic therapy were significantly lower in the positive PUAT group than in the negative PUAT group of the propensity-matched cohort.

Conclusions: We found that the sensitivity of the index test was low and specificity was high in this clinical setting. And our findings suggest that positive PUAT results may be associated with favorable clinical outcomes in patients with CO-pneumonia.

Keywords: Pneumonia; Streptococcus pneumoniae; Urinalysis; Antigens; Mortality

\section{INTRODUCTION}

Pneumonia is the leading cause of infectious disease-related deaths in adults worldwide [1]. Selecting appropriate pathogen-directed antibiotics in patients with community-onset pneumonia (CO-pneumonia) can de- crease treatment cost, drug-related adverse events, and antibiotic resistance and allow for the narrowing of empiric antibiotic therapy [2-4]. Despite the development of diagnostic techniques for pneumonia, causative organisms have not been detected in more than half of the patients [5]. Determining the etiology of pneumonia has 
remained challenging because of several reasons, including difficulties in obtaining good-quality sputum and the unreliability of subsequent culture results, low sensitivity of blood cultures, and the administration of antibiotics before sample collection $[5,6]$. Streptococcus pneumoniae is the most frequently isolated pathogen; therefore, initial empirical antibiotic treatment regimens for most patients with community-acquired pneumonia (CAP) aim at covering S. pneumoniae [3]. The early identification of $S$. pneumoniae may lead to the selection of more reliable pathogen-targeted antibiotics [3].

The pneumococcal urinary antigen test (PUAT) is an assay widely used to identify the C-polysaccharide antigen common to all serotypes excreted into the urine using a membrane immunochromatographic test [7]. In addition to the advantages of rapidity and simplicity, it has a high sensitivity and specificity over Gram stain and sputum cultures, as well as availability in patients who cannot demonstrate expectorated sputum [3]. In addition, even after antibiotic therapy has been started, the PUAT has the ability to detect pneumococcal pneumonia $[3,8]$. Current guidelines recommend that the PUAT can only be performed in patients with severe CAP or moderate- or high-severity pneumonia [3,9].

To date, the clinical benefits derived from the results of PUAT have not been fully established [10]. Thus, the aim of our study was to investigate the diagnostic performances of the PUAT, and the association between the results of PUAT and clinical outcomes in patients with CO-pneumonia.

\section{METHODS}

\section{Study design and population}

We conducted a retrospective cohort study of patients with CO-pneumonia at a university affiliated medical center. Adult patients (aged $\geq 18$ years) who were hospitalized with pneumonia between January 2012 and December 2015 were investigated. Patients were identified by the use of the international diagnostic codes version 10 to screen for possible cases as follows: J18.0 to 18.9 as representative codes of pneumonia in the primary discharge diagnosis [11]. After reviewing the medical records and radiological findings of relevant patients, we confirmed the diagnosis of pneumonia as the presence of a new infiltrate on a chest radiograph and by clinical signs [2]. We excluded the following types of patients: (1) those who were readmitted due to pneumonia within 10 days of leaving the hospital; (2) those who were transferred from other hospitals after hospitalization for > 48 hours; (3) those with obstructive pneumonia; (4) those who had immunocompromised status, such as neutropenia (absolute neutrophil count $<1,500$ cells $/ \mu \mathrm{L}$ ) after chemotherapy or human immunodeficiency virus infection; (5) those who did not receive guideline-concordant antibiotic therapy; and (6) those who had hospital-acquired pneumonia (HAP). Although the concept of healthcare-associated pneumonia (HCAP) was eliminated in the revised 2016 American Thoracic Society (ATS)/ Infectious Disease Society of America (IDSA) guidelines for the management of HAP and ventilator-acquired pneumonia [12], we included HCAP as a category of CO-pneumonia in the present study.

Urine samples were collected in the emergency department or ward in the first 24 hours to detect urinary antigens for $S$. pneumoniae using BinaxNOW (Binax Inc., Scarborough, ME, USA). According to the results of PUAT, we classified the study patients into positive and negative PUAT groups. Empiricalguideline-concordant antibiotics were maintained without regard to the results of tests. Demographics, radiological findings, laboratory findings, microbiological results, and clinical outcomes were compared between the two groups.

\section{Ethical consideration}

Ethical committees approved to review clinical data of relevant patients obtained from medical records such as clinical parameters, laboratory, radiological, and microbiological findings. Information obtained during the study was kept confidential and only intended for research purpose.

The study protocol was approved by the Ethical Review Committee of Jeju National University Hospital (Institutional Review Board no. 2018-04-001). Informed consent was waived because of the retrospective nature of the study.

\section{Microbiology and antibiotics}

Sampling to determine the microbial etiology of pneumonia included sputum, tracheobronchial aspirates, bronchoalveolar lavage fluid, pleural fluid, or blood 
through a semi-quantitative manner. The antibiotic sensitivity of all isolates was determined using a disc diffusion method. Serologic tests were performed to detect antibodies against Mycoplasma pneumoniae or Chlamydia pneumoniae. A patient was considered to have a potentially drug-resistant (PDR) pathogen if one of the following pathogens was isolated: methicillin-resistant Staphylococcus aureus (MRSA), Pseudomonas aeruginosa, extended-spectrum beta-lactamase (ESBL)-producing or carbapenem-resistant Klebsiella pneumoniae and Escherichia coli, Acinetobacter baumannii, or Stenotrophomonas maltophilia [13].

Changes in antibiotic regimens were defined as either escalation or de-escalation after culture sensitivities or clinical stabilities were identified. Inappropriate antibiotic therapy was noted if the empirical antibiotic treatment was not effective against the identified pathogen based on in vitro susceptibility testing [14]. Initial treatment failure was defined as death during initial treatment or a change of initial therapeutic agent after 48 hours due to clinical instability [15]. Guideline-concordant antibiotic therapy for CAP was defined as antibiotic regimens recommended by the 2007 ATS/IDSA guidelines for CAP: specifically, beta-lactam plus macrolide or fluoroquinolone [3]. Since the data of the present study were collected before the 2016 revised ATS/IDSA guidelines were released, guideline-concordant antibiotic therapy for HCAP was defined as antibiotic regimens recommended by the 2005 ATS/IDSA guidelines, i.e., anti-pseudomonal beta-lactam plus either a fluoroquinolone or an aminoglycoside, along with anti-MRSA coverage if risk factors were present [16].

\section{Statistical analyses}

Data are presented as median (interquartile range) for continuous variables and as a number (\%) for categorical variables. Continuous variables were compared using the Student's t test for normally distributed variables and the Mann-Whitney U test for non-normally distributed variables. Categorical variables were compared using the Pearson chi-square test, and Fisher's exact test was used when any cell contained less than five items. To evaluate the reliability of the PUAT, we calculated its sensitivity and positive and negative predictive values, using the following three different reference groups of patients as the gold standard: (1) definitive pneumococcal pneu- monias (CO-pneumonia with S. pneumoniae isolated in blood or pleural fluid culture); (2) probable pneumococcal pneumonias (CO-pneumonia with $S$. pneumoniae as the predominant morphotype on Gram stain or culture of sputum, transtracheal aspirates, and bronchoalveolar lavage); and (3) all pneumococcal pneumonias (definite plus probable) [17]. When determining specificity, we considered the control group as all patients without a diagnosis of pneumococcal pneumonia, including those with unknown etiology. We also calculated positive and negative likelihood ratios (LRs) as a measure of the extent to which the pretest odds were altered by the test results; low negative $\operatorname{LR}(<0.1)$ and high positive $\operatorname{LR}(>10)$ findings were considered to be useful for ruling out and ruling in decisions, respectively [18].

To improve the balance of baseline characteristics and reduce the effects of selection bias and potential confounding in this retrospective cohort study, estimated propensity scores were used to match the positive PUAT group to the negative PUAT group. The propensity score was calculated by logistic regression analysis using the covariates of baseline characteristics (Table 1 ). Standardized differences were estimated for all baseline covariates before and after matching to assess prematch imbalance and postmatch balance. All statistical analyses were performed using SPSS version 18.o (SPSS Inc., Chicago, IL, USA). All tests were two-sided, and $p$ values of $<0.05$ were considered to be statistically significant.

\section{RESULTS}

\section{Study population}

Fig. 1 shows patient enrolment. Overall, 1,257 patients were analyzed, of whom 163 (13.0\%) had positive PUAT results and 1,094 (87.0\%) had negative PUAT results. The proportion of patients with positive PUAT results was similar in both CAP and HCAP groups (13.1\% vs. $12.8 \%, p=0.875)$. The microbiological methods by which S. pneumoniae was identified in 115 patients as a cause of CO-pneumonia are as follows: blood cultures in seven patients (6.1\%), pleural fluid in two patients $(1.7 \%)$, transtracheal aspiration in 15 patients (13.0\%), bronchoalveolar lavage in six patients (5.2\%), and sputum in 87 patients (75.7\%). In two patients, S. pneumoniae was simultaneously identified in blood and sputum cultures, 
Table 1. Baseline characteristics of the patients

\begin{tabular}{|c|c|c|c|c|c|c|}
\hline \multirow[b]{2}{*}{ Characteristic } & \multicolumn{3}{|c|}{ Entire cohort $(n=1,257)$} & \multicolumn{3}{|c|}{ Propensity score-matched cohort $(n=322)$} \\
\hline & $\begin{array}{l}\text { Positive PUAT } \\
(\mathrm{n}=163)\end{array}$ & $\begin{array}{l}\text { Negative PUAT } \\
\quad(n=1,094)\end{array}$ & $p$ value & $\begin{array}{l}\text { Positive PUAT } \\
\quad(\mathrm{n}=161)\end{array}$ & $\begin{array}{l}\text { Negative PUAT } \\
\quad(n=161)\end{array}$ & $p$ value \\
\hline Age, yr & $73(66-81)$ & $72(61-80)$ & 0.124 & $73(66-81)$ & $74(65-81)$ & 0.683 \\
\hline Male sex & $105(64.4)$ & $673(61.5)$ & 0.477 & $103(64.0)$ & $111(68.9)$ & 0.345 \\
\hline Tube feeding & $6(3.7)$ & $45(4 \cdot 1)$ & 0.794 & $6(3 \cdot 7)$ & $6(3 \cdot 7)$ & 1.000 \\
\hline $\begin{array}{l}\text { Hospitalization for } 2 \text { or more days } \\
\text { in the past } 90 \text { days }\end{array}$ & $25(15 \cdot 3)$ & $207(18.9)$ & 0.271 & $25(15 \cdot 5)$ & $29(18.0)$ & $0.55^{1}$ \\
\hline $\begin{array}{l}\text { Residence in a nursing home or } \\
\text { long-term care facility }\end{array}$ & $30(18.4)$ & $139(12.7)$ & 0.047 & $30(18.6)$ & $29(18.0)$ & 0.885 \\
\hline $\begin{array}{l}\text { Recent outpatient intravenous } \\
\text { therapy } \leq 30 \text { days }\end{array}$ & $6(3.7)$ & $39(3.6)$ & 0.941 & $6(3.7)$ & $4(2.5)$ & $0.55^{1}$ \\
\hline $\begin{array}{l}\text { Attendance at a dialysis center in } \\
\text { the previous } 30 \text { days }\end{array}$ & $1(0.6)$ & $24(2.2)$ & 0.238 & $1(0.6)$ & $2(1.2)$ & 1.000 \\
\hline \multicolumn{7}{|l|}{ Comorbidity } \\
\hline Malignancy $^{\mathrm{a}}$ & $29(17.8)$ & $197(18.0)$ & 0.947 & $28(17 \cdot 4)$ & $27(16.8)$ & 0.882 \\
\hline Chronic liver disease $\mathrm{b}^{\mathrm{b}}$ & $10(6.1)$ & $54(4 \cdot 9)$ & 0.516 & $10(6.2)$ & $11(6.8)$ & 0.821 \\
\hline Chronic heart disease $^{c}$ & $23(14.1)$ & $169(15 \cdot 4)$ & 0.658 & $23(14 \cdot 3)$ & $19(11.8)$ & 0.508 \\
\hline Chronic kidney disease $^{\mathrm{d}}$ & $11(6.7)$ & $126(11.5)$ & 0.068 & $11(6.8)$ & $16(9.9)$ & 0.315 \\
\hline Diabetes mellitus ${ }^{\mathrm{e}}$ & $41(25 \cdot 2)$ & $251(22.9)$ & 0.533 & $40(24.8)$ & $48(29.8)$ & 0.317 \\
\hline Chronic respiratory disease $^{f}$ & $38(23 \cdot 3)$ & $295(27.0)$ & 0.324 & $38(23.6)$ & $38(23.6)$ & 1.000 \\
\hline Central nervous system disorders ${ }^{\mathrm{g}}$ & $49(30.1)$ & $264(24.1)$ & 0.102 & $48(29.8)$ & $54(33 \cdot 5)$ & 0.472 \\
\hline Two or more comorbidities & $60(36.8)$ & $436(39 \cdot 9)$ & $0.45^{8}$ & $59(36.6)$ & $66(41.0)$ & 0.423 \\
\hline \multicolumn{7}{|l|}{ Clinical parameters } \\
\hline Altered mental state ${ }^{\mathrm{h}}$ & $15(9.2)$ & $100(9.1)$ & 0.980 & $15(9 \cdot 3)$ & $14(8.7)$ & 0.846 \\
\hline Respiratory failure ${ }^{i}$ & $58(35 \cdot 6)$ & $359(32.8)$ & 0.484 & $57(35 \cdot 4)$ & $62(38.5)$ & 0.564 \\
\hline Sepsis or septic shock at onset ${ }^{j}$ & $22(13 \cdot 5)$ & $138(12.6)$ & 0.752 & $22(13.7)$ & $23(14 \cdot 3)$ & 0.872 \\
\hline Severe pneumonia & $26(16.0)$ & $165(15 \cdot 1)$ & 0.773 & $26(16.1)$ & $27(16.8)$ & 0.881 \\
\hline Intensive care unit admission & $21(12.9)$ & $132(12.1)$ & 0.745 & $20(12.4)$ & $21(13.0)$ & 0.867 \\
\hline Need for ventilation & $8(4 \cdot 9)$ & $69(6.3)$ & 0.487 & $8(5 \cdot 0)$ & $10(6.2)$ & 0.628 \\
\hline \multicolumn{7}{|l|}{ Radiological findings } \\
\hline Multi-lobar involvement & $86(52.8)$ & $504(46.1)$ & 0.127 & $84(52.2)$ & $83(51.6)$ & 0.921 \\
\hline Pleural effusion & $34(20.9)$ & $189(17 \cdot 3)$ & 0.264 & $32(19.9)$ & $31(19 \cdot 3)$ & 0.888 \\
\hline \multicolumn{7}{|l|}{ Laboratory findings } \\
\hline White blood cells, $/ \mathrm{mm}^{3}$ & $\begin{array}{c}11,600 \\
(8,600-16,100)\end{array}$ & $\begin{array}{c}10,600 \\
(7,675-14,100)\end{array}$ & 0.011 & $\begin{array}{c}11,600 \\
(8,500-16,000)\end{array}$ & $\begin{array}{c}11,800 \\
(8,650-15,850)\end{array}$ & 0.877 \\
\hline C-reactive protein, mg/dL & $12.0(5.9-18.9)$ & $9 \cdot 7(4.2-17.2)$ & 0.020 & $12.0(5.8-18.7)$ & $13.3(6.3-21.7)$ & 0.432 \\
\hline \multicolumn{7}{|l|}{ Indices for disease severity } \\
\hline CURB-65 score & $1(1-2)$ & $1(1-2)$ & 0.054 & $1(1-2)$ & $1(1-2)$ & 0.737 \\
\hline PSI score & $98(76-123)$ & $92(70-121)$ & 0.080 & $98(76-123)$ & $99(76-131)$ & 0.610 \\
\hline
\end{tabular}

Values are presented as median (interquartile range) or number (\%). Septic shock was defined as sepsis with persisting hypotension requiring vasopressors to maintain a mean arterial pressure of $\geq 65 \mathrm{mmHg}$ and having a serum lactate level of $>2$ $\mathrm{mmol} / \mathrm{L}(18 \mathrm{mg} / \mathrm{dL})$ despite adequate volume resuscitation.

PUAT, pneumococcal urinary antigen test; CURB-65, confusion, urea, respiratory rate, blood pressure, age $\geq 65$ years; MRSA, methicillin-resistant Staphylococcus aureus; PSI, pneumonia severity index. 


\section{Table 1. Continued}

${ }^{\text {a }}$ Malignancy includes active at the time of presentation or requiring anticancer treatment within the previous 5 years.

${ }^{\mathrm{b}}$ Chronic liver disease included preexisting viral or toxic hepatopathy at the time of pneumonia diagnosis.

${ }^{\mathrm{c}}$ Chronic heart disease was identified based on past history or administration of diuretics for the treatment of heart disease.

${ }^{\mathrm{d}}$ Chronic kidney disease included preexisting renal disease with documented abnormal serum creatinine levels.

${ }^{\mathrm{e}}$ Diabetes mellitus included a history of diagnosis of intolerance to glucose, hemoglobin Aic $\geq 6.5 \%$, or treatment with oral hypoglycemic agents or insulin.

${ }^{\mathrm{f}}$ Chronic respiratory disease included simple chronic bronchitis, chronic obstructive pulmonary disease, and structural lung diseases such as bronchiectasis and interstitial lung diseases.

${ }^{g}$ Central nervous system disorders included acute or chronic vascular or nonvascular encephalopathy with or without dementia.

${ }^{\mathrm{h}}$ Altered mental state was defined as Glasgow Coma Score $\leq 13$ documented by the physician.

${ }^{\mathrm{i}}$ Respiratory failure was defined when partial pressure of oxygen in arterial blood $\left(\mathrm{PaO}_{2}\right)$ was $60 \mathrm{mmHg}$ or less or when the $\mathrm{PaO} 2 /$ fraction of inspired oxygen $(\mathrm{FiO} 2)$ ratio was $300 \mathrm{mmHg}$ or less.

${ }^{j}$ Sepsis was defined as organ dysfunction identified as an acute change in the Sequential Organ Failure Assessment score of $\geq 2$ consequent to pneumonia.

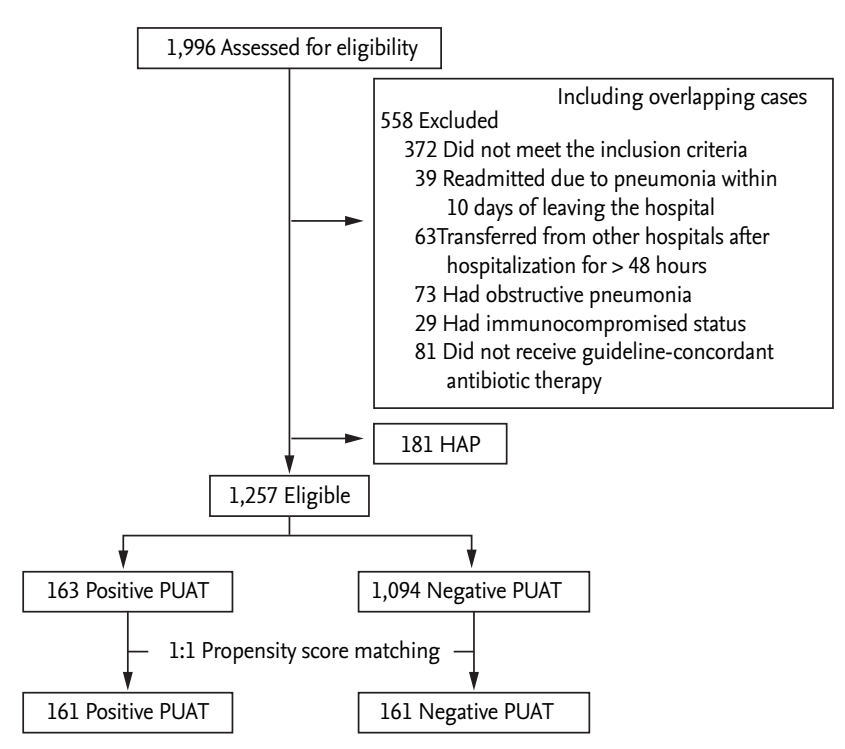

Figure 1. Flow diagram of patient enrollment. HAP, hospital-acquired pneumonia; PUAT, pneumococcal urinary antigen test.

and PUAT was positive in seven of nine (77.8\%) patients with definitive pneumococcal pneumonia. Also, in 108 patients with probable pneumococcal pneumonia, 58 (53.7\%) tested positive. Taking into account both of the groups together (i.e., all pneumococcal pneumonia), the results of PUAT was positive in 65 patients (56.5\%).

Baseline characteristics are presented in Table 1. Prior to matching, patients in the positive PUAT group were more likely to reside in a nursing home or long-term care facility. In addition, the median white blood cell and C-reactive protein levels were higher in the positive PUAT group than in the negative PUAT group. The propensity score-matching process provided 161 pairs of patients with positive and negative PUAT findings and achieved a good balance for all baseline comorbidities, clinical parameters, and severity indexes.

\section{Diagnostic accuracy of the PUAT in patients with CO-pneumonia}

Table 2 shows the calculated values of sensitivity, specificity, positive and negative predictive values, and positive and negative LRs by the different methods. In patients with all pneumococcal pneumonia, the sensitivity of PUAT was $56.5 \%$ ( $95 \%$ confidence interval [CI], 47.0 to 65.7) and the specificity was $90.9 \%$ (95\% CI, 89.0 to 92.6). Additionally, the positive predictive value was $39.9 \%$ (95\% CI, 34.1 to 46.0) and the negative predictive value was 95.1\% (95\% CI, 94.1 to 96.0). Positive and negative LRs were 6.2 (95\% CI,4.9 to 8.0) and 0.5 (95\% CI, 0.4 to o.6).

\section{Microorganisms}

Table 3 shows the distributions of microorganisms on the basis of the culture results. Overall etiology was established in 97 (59.5\%) and 518 (47.3\%) patients in the positive PUAT and the negative PUAT groups, respectively. Among patients with positive PUAT results, other microorganisms were identified in $36(22.1 \%)$ patients using conventional methods. In both the full and propensity-matched cohorts, the rate of $S$. pneumoniae identified was significantly higher in the positive PUAT group $39.9 \%$ vs. $4.6 \%, p<0.001$ and $39.5 \%$ vs. $4.3 \%, p<$ 
Table 2. Diagnostic accuracy of the PUAT in patients with community-onset pneumonia

\begin{tabular}{lcccccc}
\hline $\begin{array}{l}\text { Diagnosis of } \\
\text { pneumococcal } \\
\text { pneumonia }\end{array}$ & Sensitivity & Specificity & Positive PV & Negative PV & Positive LR & Negative LR \\
\hline $\begin{array}{l}\text { Definitive } \\
\text { Probable }\end{array}$ & $77.8(40.0-97.2)$ & $87.3(85.4-89.1)$ & $4.2(3.0-6.1)$ & $99.8(99.4-100)$ & $6.1(4.2-9.0)$ & $0.3(0.1-0.9)$ \\
Overall & $53.7(43.9-63.3)$ & $90.9(89.1-92.5)$ & $35.6(30.0-41.6)$ & $95.4(94.5-96.2)$ & $5.9(4.6-7.6)$ & $0.5(0.4-0.6)$ \\
\hline
\end{tabular}

Values are presented as $95 \%$ confidence interval.

PUAT, pneumococcal urinary antigen test; PV, predictive value; LR, likelihood ratio.

Table 3. Microorganisms isolated from the patients on the basis of culture results

\begin{tabular}{|c|c|c|c|c|c|c|}
\hline \multirow[b]{2}{*}{ Variable } & \multicolumn{3}{|c|}{ Entire cohort $(\mathrm{n}=1,257)$} & \multicolumn{3}{|c|}{ Propensity score-matched cohort $(\mathrm{n}=322)$} \\
\hline & $\begin{array}{c}\text { Positive PUAT } \\
(\mathrm{n}=163)\end{array}$ & $\begin{array}{l}\text { Negative PUAT } \\
(\mathrm{n}=1,094)\end{array}$ & $p$ value & $\begin{array}{l}\text { Positive PUAT } \\
(\mathrm{n}=161)\end{array}$ & $\begin{array}{l}\text { Negative PUAT } \\
\quad(\mathrm{n}=161)\end{array}$ & $p$ value \\
\hline \multicolumn{7}{|l|}{ Microorganisms $^{\mathrm{a}}$} \\
\hline Identified pathogens & $97(59 \cdot 5)$ & $518(47 \cdot 3)$ & 0.004 & $95(59.0)$ & $85(52.8)$ & 0.262 \\
\hline \multicolumn{7}{|l|}{ Gram-positive bacteria } \\
\hline Streptococcus pneumoniae & $65(39 \cdot 9)$ & $50(4.6)$ & $<0.001$ & $64(39.8)$ & $7(4 \cdot 3)$ & $<0.001$ \\
\hline Staphylococcus aureus & $2(1.2)$ & $43(3.9)$ & 0.083 & $2(1.2)$ & $9(5.6)$ & 0.032 \\
\hline Methicillin-sensitive $S$. aureus & o & $20(1.8)$ & 0.096 & o & $6(3.7)$ & 0.030 \\
\hline Methicillin-resistant S. aureus & $2(1.2)$ & $23(2.1)$ & 0.762 & $2(1.2)$ & $3(1.9)$ & 1.000 \\
\hline Other Gram-positive bacteria & $\mathrm{o}(\mathrm{o})$ & $17(1.6)$ & 0.151 & o & $2(1.2)$ & 0.498 \\
\hline \multicolumn{7}{|l|}{ Gram-negative bacteria } \\
\hline Pseudomonas aeruginosa & $8(4 \cdot 9)$ & $46(4.2)$ & 0.680 & $8(5.0)$ & $6(3.7)$ & 0.585 \\
\hline Haemophilus influenza & $1(0.6)$ & $13(1.2)$ & 1.000 & $1(0.6)$ & $1(0.6)$ & 0.000 \\
\hline Klebsiella pneumoniae & $9(5 \cdot 5)$ & $69(6.3)$ & 0.698 & $8(5.0)$ & $11(6.8)$ & 0.478 \\
\hline ESBL $(+)$ & $1(0.6)$ & $8(0.7)$ & 1.000 & $1(0.6)$ & $1(0.6)$ & 1.000 \\
\hline $\operatorname{ESBL}(-)$ & $8(4 \cdot 9)$ & $61(5.6)$ & 0.727 & $7(11.5)$ & $10(6.2)$ & 0.455 \\
\hline Acinetobacter species & $\mathrm{O}$ & $11(1.0)$ & 0.377 & 0 & $4(2.5)$ & 0.123 \\
\hline Other Gram-negative species ${ }^{\mathrm{b}}$ & $2(1.2)$ & $14(1.3)$ & 1.000 & $2(1.2)$ & $1(0.6)$ & 1.000 \\
\hline Mycoplasma pneumonia & $14(8.6)$ & $61(5.6)$ & 0.130 & $14(8.7)$ & $9(5 \cdot 6)$ & 0.279 \\
\hline
\end{tabular}

Values are presented as number (\%). Percentages refer to the division by the total number of patients.

PUAT, pneumococcal urinary antigen test; ESBL, extended-spectrum $\beta$-lactamase.

${ }^{a}$ Allowed for overlapping.

${ }^{\mathrm{b}}$ Other Gram-negative species included Escherichia coli, Enterobacter species, Serratia marcescens, and Legionella pneumophilia.

0.001). Following propensity score matching, the rate of Staphylococcus aureus was statistically higher in the negative PUAT group (1.2\% vs. 5.6\%, $p=0.032$ ).

\section{Associations between the PUAT and clinical out- comes}

The primary and secondary study outcomes are summarized in Table 4. In the full cohort of 1,257 patients, there were no significant differences in 30-day mortality between groups $(6.1 \%$ in the positive PUAT group vs. $8.2 \%$ in the negative PUAT group; odds ratio [OR], 0.73 ; 95\% CI, 0.37 to 1.43; $p=0.357$ ) (Fig. $2 \mathrm{~A}$ ). However, in the propensity-matched cohort, the 30-day mortality rates were lower in the positive PUAT group when compared with the negative PUAT group (5.6\% vs. 17.4\%; OR, 0.28; 95\% CI, 0.13 to $0.62 ; p=0.001$ ) (Fig. $2 \mathrm{~B}$ ). In subgroup 
Table 4. The associations between the PUAT and clinical outcomes before and after propensity score matching

\begin{tabular}{|c|c|c|c|c|}
\hline Outcomes & Positive PUAT & Negative PUAT & $p$ value & Effect size \\
\hline \multicolumn{5}{|l|}{ Primary outcome } \\
\hline \multicolumn{5}{|l|}{ 30-day mortality, \% } \\
\hline Crude (full cohort) & 6.1 & 8.2 & 0.357 & $0.73(0.37 \text { to } 1.43)^{\mathrm{a}}$ \\
\hline Propensity score matching ${ }^{b}$ & 5.6 & $17 \cdot 4$ & 0.001 & $0.28(0.13 \text { to } 0.62)^{\mathrm{a}}$ \\
\hline \multicolumn{5}{|l|}{ Secondary outcomes } \\
\hline \multicolumn{5}{|l|}{ PDR pathogens ${ }^{c}, \%$} \\
\hline Crude (full cohort) & 6.7 & 9.8 & 0.216 & $0.67(0.35 \text { to } 1.27)^{\mathrm{a}}$ \\
\hline Propensity score matching ${ }^{b}$ & $4 \cdot 3$ & 10.6 & 0.034 & $0.39(0.16 \text { to } 0.96)^{a}$ \\
\hline \multicolumn{5}{|l|}{ Duration of antibiotic therapy, day } \\
\hline Crude (full cohort) & $11.1 \pm 6.0$ & $11.6 \pm 8.2$ & 0.590 & $-0.50(-1.54 \text { to } 0.54)^{\mathrm{d}}$ \\
\hline Propensity score matching ${ }^{b}$ & $11.1 \pm 6.0$ & $11.9 \pm 6.7$ & 0.294 & $-0.80(-2.19 \text { to } 0.59)^{\mathrm{d}}$ \\
\hline \multicolumn{5}{|l|}{ Change of antibiotics, \% } \\
\hline Crude (full cohort) & 17.8 & 23.0 & 0.134 & $0.72(0.47 \text { to } 1.11)^{a}$ \\
\hline Propensity score matching ${ }^{b}$ & $17 \cdot 4$ & 26.7 & 0.044 & $0.58(0.34 \text { to } 0.99)^{\mathrm{a}}$ \\
\hline \multicolumn{5}{|l|}{ Use of inappropriate antibiotics, \% } \\
\hline Crude (full cohort) & 6.1 & 6.7 & 0.796 & $0.91(0.46 \text { to } 1.81)^{\mathrm{a}}$ \\
\hline Propensity score matching ${ }^{\mathrm{b}}$ & 5.6 & 6.2 & 0.813 & $0.89(0.35 \text { to } 2.26)^{\mathrm{a}}$ \\
\hline \multicolumn{5}{|c|}{ Failure of initial antibiotic therapy, \% } \\
\hline Crude (full cohort) & $15 \cdot 3$ & 21.5 & 0.071 & $0.66(0.42 \text { to } 1.04)^{\mathrm{a}}$ \\
\hline Propensity score matching ${ }^{\mathrm{b}}$ & 14.9 & 30.0 & 0.004 & $0.45(0.26 \text { to } 0.79)^{\mathrm{a}}$ \\
\hline \multicolumn{5}{|l|}{ Length of hospital stay, day } \\
\hline Crude (full cohort) & $10.8 \pm 12.0$ & $9.6 \pm 8.8$ & 0.737 & $1.20(-0.71 \text { to } 3.11)^{\mathrm{d}}$ \\
\hline Propensity score matching ${ }^{\mathrm{b}}$ & $10.9 \pm 12.1$ & $12.3 \pm 10.5$ & 0.153 & $-1.40(-3.87 \text { to } 1.07)^{\mathrm{d}}$ \\
\hline
\end{tabular}

Values are presented as percentage or mean $\pm \mathrm{SD}$.

PUAT, pneumococcal urinary antigen test; PDR, potentially drug-resistant; CURB-65, confusion, urea, respiratory rate, blood pressure, age $\geq 65$ years; ESBL, extended-spectrum $\beta$-lactamase.

${ }^{\mathrm{a}}$ Odds ratio (95\% confidence interval).

${ }^{\mathrm{b}}$ The variables included as covariates in the propensity score matching were age; sex; tube feeding; comorbidities (e.g., malignancy, chronic liver disease, chronic heart disease, chronic kidney disease, diabetes mellitus, chronic respiratory disease, central nervous system disorders, and two or more comorbidities); altered mental state; respiratory failure; sepsis or septic shock; intensive care unit admission; need for ventilation; antibiotic use before admission; multi-lobar involvement; pleural effusion; white blood cells; C-reactive protein; CURB-65 score; and pneumonia severity index.

${ }^{\mathrm{c}}$ PDR pathogens included methicillin-resistant Staphylococcus aureus, Pseudomonas species, Acinetobacter species, Stenotrophomonas maltophilia, and ESBL-producing Enterobacteriaceae.

${ }^{\mathrm{d}}$ Mean difference (95\% confidence interval).

analysis for overall patients with severe pneumonia, the 30-day mortality was not significantly different between the positive and negative PUAT groups (23.1\% vs. 33.1\%, $p=0.297)$. On the other hand, in the propensity-matched cohort of severe pneumonia, the 30-day mortality rates were lower in the positive PUAT group (23.1\% vs. $59.3 \%$, $p=0.008$ ).
In the full cohort, there were no significant differences in all secondary study outcomes between groups. In the propensity-matched cohort, the proportion of patients with PDR pathogens was significantly lower in the positive PUAT group (4.3\% vs. 10.6\%; OR, 0.39; 95\% CI, 0.16 to $0.96 ; p=0.034$ ). Likewise, the proportion of patients with a change of antibiotics and failure of initial 

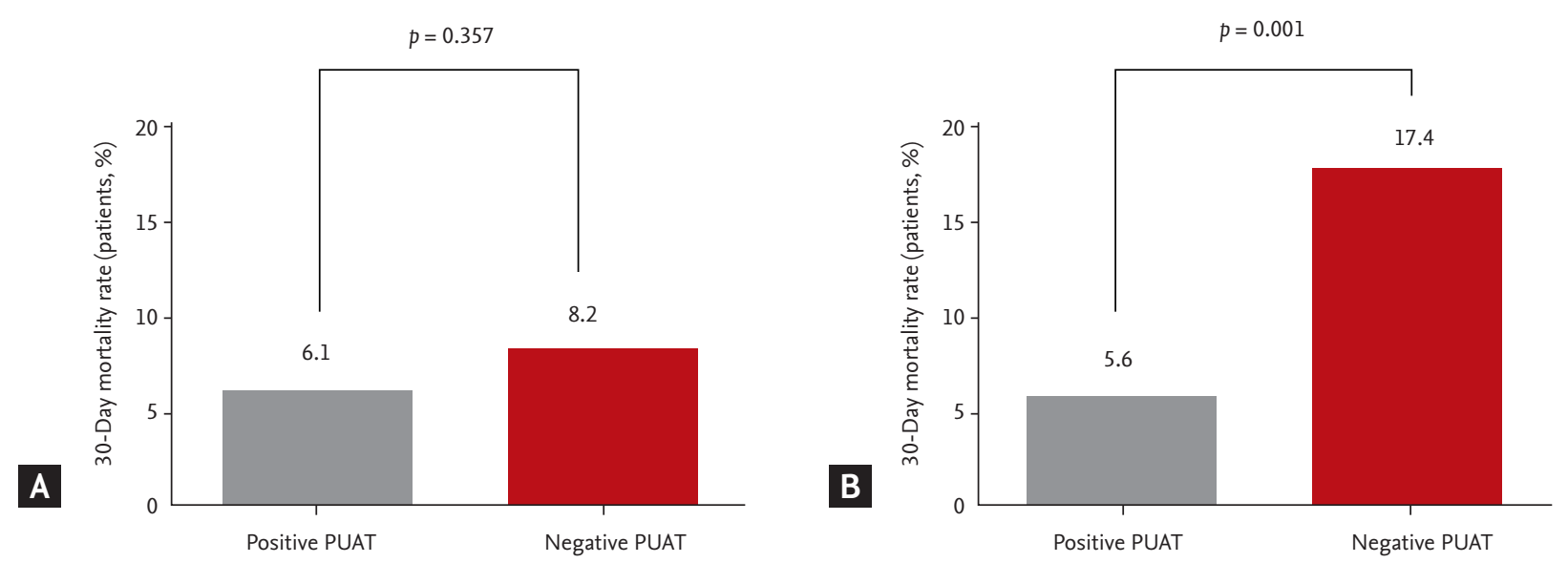

Figure 2. Associations between the pneumococcal urinary antigen test (PUAT) and 30-day mortality rate. (A) The full cohort and (B) the propensity score-matched cohort.

antibiotic therapy was also statistically lower in the positive PUAT group compared to the negative PUAT group ([17.4\% vs. 26.7\%; OR, 0.58; 95\% CI, 0.34 to $0.99 ; p=0.044]$ and $[14.9 \%$ vs. $30.0 \%$; OR, $0.45 ; 95 \% \mathrm{CI}, 0.26$ to $0.79 ; p=$ ०.004], respectively).

\section{DISCUSSION}

In our study, we identified three major findings. First, approximately $13 \%$ of patients with CO-pneumonia had positive PUAT results, and the sensitivity and specificity values for the diagnosis of definitive, probable, and overall pneumococcal pneumonia were $77.8 \%$ and $87.3 \%$, $53.7 \%$, and $90.9 \%$, and $56.5 \%$ and $90.9 \%$, respectively. Second, the clinical parameters of the positive PUAT group were not worse than in the negative PUAT group. Third, in the propensity-matched cohort, the 30-day mortality rates were lower in the positive PUAT group.

In patients with CO-pneumonia, the selection of empirical antibiotic regimen is based on the prediction of the most common pathogens [3,9]. Since the widespread use of pneumococcal conjugate vaccines was initiated, recent studies have reported a decline in $S$. pneumoniae as a cause of CAP $[2,19,20]$. However, $S$. pneumoniae is still the most common cause at $5 \%$ to $21 \%$ among microorganisms leading to hospitalization in patients with CAP [7,21-23]. The PUAT is simple, rapid, and useful for detecting pneumococcal pneumonia when samples for culture cannot be obtained in a timely fashion or when antibiotic therapy has already commenced $[3,8]$. Previously, a meta-analysis demonstrated that the pooled sensitivity and specificity of the PUAT for definitive pneumococcal pneumonia in the same clinical setting used in the present study were $75 \%$ and $80 \%$, respectively [24]. Our results indicated that the diagnostic performances of the PUAT for definitive pneumococcal pneumonia were comparable with the results of a meta-analysis [24].

The PUAT has been reported to have a higher diagnostic yield in patients with severe pneumonia [25]. Although a previous study revealed that the PUAT was more sensitive in patients with high-risk pneumonia as compared to those without (94\% vs. 63\%, $p<0.001$ ) [25], the rate of severe pneumonia in our study was similar between both groups (16.0\% vs. $15.1 \%, p=0.773)$. And, while a recent multicenter USA study reported that patients with HCAP were less likely to have positive PUAT results than those with CAP [10], there were no differences in positive PUAT results between the CAP and HCAP groups in our study.

The association between PUAT result and clinical outcomes in patients with CO-pneumonia has been investigated in a few studies $[26,27]$. A large prospective study further demonstrated that positive PUAT results were significantly associated with 30-day mortality, intensive care unit admission, use of mechanical ventilation, treatment failure, and adverse outcomes in patients with bacteremic pneumococcal pneumonia [26]. However, this study contained the possibility of selection bias, and patients with positive PUAT results were 
significantly worse in clinical parameters such as respiratory rates, arterial oxygen pressure, and $\mathrm{pH}$ levels, and multi-lobar involvement [26]. In the present study, we found that 30-day mortality rates were significantly lower in the positive PUAT group after adjustment for initial presentations using the propensity score-matching process. Similarly, in a recent registry-based retrospective study using a modified CRB65 (confusion, respiratory rate, blood pressure, and age $\geq 65$ years) score by subtracting 1 from the scoring system if the PUAT was positive, positive PUAT results were associated with lower risk of 30-day mortality [27]. We additionally investigated the association between the PUAT results and secondary outcomes. In the propensity-matched cohort, the proportion of patients with PDR pathogens, changes in antibiotics, and failure rates of initial antibiotic therapy were significantly higher in the negative PUAT group than in the positive PUAT group.

Reasons for why PUAT is negative in some patients with pneumococcal pneumonia have been proposed to include the following: low levels of C-polysaccharide antigen and sequestration of the antigen by binding to serum antibodies in immune complexes, reduced urinary excretion of the antigen, delayed times from the onset of symptoms to diagnosis, and previous antibiotic treatment [26]. We tried to find clinical parameters of false negative PUAT results. Compared to patients with true positive PUAT results, those with false negative PUAT results had chronic heart diseaseless frequently $(18.5 \%$ vs. $8.0 \%, p=0.049)$. However, because of the small sample size $(n=165)$ and low statistical power $(p=0.049)$, we could not allow for this as a crucial parameter.

There remains controversy with regard to the change of antibiotics on the basis of the results of PUAT [28,29]. In a prospective study including 219 patients with non-severe CAP, the targeted use of amoxicillin based on PUAT results was able to decrease antibiotic resistance and reduce the use of unnecessary antibiotics [28]. In contrast, another randomized controlled trial reported that $S$. pneumoniae-targeted therapy with positive PUAT results increased the rate of clinical relapse compared with empirical therapy [29]. Possible explanations for treatment failure may include the following: first, the possibility of polymicrobial infections cannot be excluded [29]. Especially in the case of polymicrobial infections with PDR pathogens, narrowing the anti- biotic treatment can lead to treatment failure. Second, a high proportion of pathogens remain unidentified in patients with CO-pneumonia. This means that it is difficult to predict the rate of polymicrobial infections. Third, pneumococcal capsular polysaccharides could react with othermicroorganisms such as Staphylococcus, Streptococcus species, and Gram-negative strains [30]. Other microorganisms identified in patients with positive PUAT results through conventional methods, might be regarded as having cross-reactivity [30]. Possible cross-reacting microorganisms could elicit treatment failure [30]. Finally, the presence of drug-resistant S. pneumoniae may also be a plausible explanation [28]. The emergence and spread of drug-resistant $S$. pneumoniae have been reported in recent years, and antibiotic resistance threatens the successful management of pneumococcal pneumonia [31]. In a meta-analysis encompassing 10 studies that involved 3,430 patients, the combined relative risks of all-cause mortality for the penicillin-resistant S. pneumoniae groups was 1.29 (95\% CI, 1.01 to 1.66) when compared with the penicillin-susceptible S. pneumoniae group [32].

In principle, patients with positive PUAT results would have a good response to empirical guideline-concordant antibiotic therapy, which has been associated with a significant decrease in in-hospital mortality, length of hospital stay, and the duration of parenteral therapy [33]. We maintained guideline-concordant empirical antibiotics without regard to the results of tests and investigated the association between the PUAT results and clinical outcomes in the present study.

The strength of our study is in the propensity scorematching process, although our subjects were a retrospective cohort. Meanwhile, there are some study limitations. First, because our study was conducted retrospectively involving patients admitted to a single institution, our data should be interpreted with caution. In particular, even though pneumococcal vaccination might produce a false positive result in the PUAT in clinical practice [34], we could not identify the rate of pneumococcal vaccination due to the retrospective nature of the study. Second, we did not perform viral testing in most of the included patients. Mixed infections consisted of approximately $5 \%$ to $10 \%$ of the etiology for CAP in previous studies $[20,27]$. Most polymicrobial agents in these studies were viral pathogens, which do not usually require specific anti- 
microbial therapy $[20,35]$. Because we did not perform routine viral testing, the role of viral agents in CO-pneumonia might be underestimated in clinical outcomes. Third, previous studies have reported the relationship between serotype of $S$. pneumoniae and disease outcome [36]. A pooled estimates for invasive pneumococcal disease demonstrated that serotypes $1,7 \mathrm{~F}$, and 8 were associated with decreased risk ratios, while serotypes 3, 6A, $6 \mathrm{~B}, 9 \mathrm{~N}$, and ${ }_{19} \mathrm{~F}$ were associated with increased risk ratios [36]. However, the serotype of $S$. pneumoniae was not investigated in this clinical setting.

In conclusion, our study revealed that the PUAT had low sensitivity and high specificity for overall pneumococcal pneumonia. After baseline characteristics were adjusted through the propensity score-matching process, empirical guideline-concordant antibiotic therapy in CO-pneumonia was associated with a lower rate of 30-day mortality in patients with positive PUAT results.

\section{KEY MESSAGE}

1. The pneumococcal urinary antigen test (PUAT) had low sensitivity and high specificity for overall pneumococcal pneumonia.

2. Baseline clinical parameters were similar between the positive PUAT and negative PUAT groups.

3. In the propensity-matched cohort, the 30-day mortality rates were lower in the positive PUAT group.

\section{Conflict of interest}

No potential conflict of interest relevant to this article was reported.

\section{Acknowledgments}

This work was supported by a research grant from Jeju National University Hospital in 2018.

\section{REFERENCES}

1. Zar HJ, Madhi SA, Aston SJ, Gordon SB. Pneumonia in low and middle incomecountries: progress and challenges. Thorax 2013;68:1052-1056.
2. Musher DM, Thorner AR. Community-acquired pneumonia. N Engl J Med 2014;371:1619-1628.

3. Mandell LA, Wunderink RG, Anzueto A, et al. Infectious Diseases Society of America/American Thoracic Society consensus guidelines on themanagement of community-acquired pneumonia in adults. Clin Infect Dis 2007;44:S27-S72.

4. van der Eerden MM, Vlaspolder F, de Graaff CS, et al. Comparison between pathogen directed antibiotic treatment andempirical broad spectrum antibiotic treatment in patients with community acquired pneumonia: a prospective randomised study. Thorax 2005;60:672-678.

5. Garcia-Vazquez E, Marcos MA, Mensa J, et al. Assessment of the usefulness of sputum culture for diagnosis of community-acquired pneumonia using the PORT predictive scoring system. Arch Intern Med 2004;164:1807-1811.

6. Campbell SG, Marrie TJ, Anstey R, Dickinson G, Ackroyd-Stolarz $\mathrm{S}$. The contribution of blood cultures to the clinical management of adult patients admitted to the hospital with community-acquired pneumonia: a prospective observational study. Chest 2003;123:1142-1150.

7. Molinos L, Zalacain R, Menendez R, et al. Sensitivity, specificity, and positivity predictors of the pneumococcal urinary antigen testin community-acquired pneumonia. Ann Am Thorac Soc 2015;12:1482-1489.

8. Smith MD, Derrington P, Evans R, et al. Rapid diagnosis of bacteremic pneumococcal infections in adults by using the Binax NOW Streptococcus pneumoniae urinary antigen test: a prospective, controlled clinical evaluation. J Clin Microbiol 2003;41:2810-2813.

9. Lim WS, Baudouin SV, George RC, et al. BTS guidelines for themanagement of community acquired pneumonia in adults: update 2009. Thorax 2009;64:iii1-iii55.

10. West DM, McCauley LM, Sorensen JS, Jephson AR, Dean NC. Pneumococcal urinary antigen test use in diagnosis and treatment of pneumonia in seven Utah hospitals. ERJ Open Res 2016;2:000112016.

11. Dean NC, Jones BE, Jones JP, et al. Impact of an electronic clinical decision support tool for emergency department patients with pneumonia. Ann Emerg Med 2015;66:511520.

12. Kalil AC, Metersky ML, Klompas M, et al. Management of adults with hospital-acquired and ventilator-associated pneumonia: 2016 clinical practice guidelines by the Infectious Diseases Society of Americaand the American Thoracic Society. Clin Infect Dis 2016;63:e61-e111. 
13. Shindo Y, Sato S, Maruyama E, et al. Health-care-associated pneumonia among hospitalized patients in a Japanese community hospital. Chest 2009;135:633-640.

14. Micek ST, Kollef KE, Reichley RM, Roubinian N, Kollef MH. Healthcare-associated pneumonia and community-acquired pneumonia: a single-centerexperience. Antimicrob Agents Chemother 2007;51:3568-3573.

15. Yamagishi Y, Mikamo H. A retrospective study of health care-associatedpneumonia patients at Aichi Medical University hospital. J Infect Chemother 2011;17:756-763.

16. American Thoracic Society; Infectious Diseases Society of America. Guidelinesfor the management of adults with hospital-acquired, ventilator-associated, andhealthcare-associated pneumonia. Am J Respir Crit Care Med 2005;171:388-416.

17. Sorde R, Falco V, Lowak M, et al. Current and potential usefulness of pneumococcal urinary antigen detection in hospitalized patients with community-acquired pneumonia toguide antimicrobial therapy. Arch Intern Med 2011;171:166-172.

18. Deeks JJ, Altman DG. Diagnostic tests 4: likelihood ratios. BMJ 2004;329:168-169.

19. Gadsby NJ, Russell CD, McHugh MP, et al. Comprehensive molecular testing for respiratory pathogens in community-acquired pneumonia. Clin Infect Dis 2016;62:817-823.

20. Johansson N, Kalin M, Tiveljung-Lindell A, Giske CG, Hedlund J. Etiology of community-acquired pneumonia: increased microbiological yield with new diagnostic methods. Clin Infect Dis 2010;50:202-209.

21. Postma DF, van Werkhoven CH, van Elden LJ, et al. Antibiotic treatment strategies for community-acquired pneumonia in adults. N Engl J Med 2015;372:1312-1323.

22. Jain S, Self WH, Wunderink RG; CDC EPIC Study Team. Community-acquired pneumonia requiring hospitalization. N Engl J Med 2015;373:2382.

23. Webb BJ, Dascomb K, Stenehjem E, Dean N. Predicting risk of drug-resistant organisms in pneumonia: moving beyond the HCAP model. Respir Med 2015;109:1-10.

24. Horita N, Miyazawa N, Kojima R, et al. Sensitivity and specificity of the Streptococcus pneumoniae urinary antigen test for unconcentrated urine from adult patients with pneumonia: a meta-analysis. Respirology 2013;18:1177-1183.

25. Roson B, Fernandez-Sabe N, Carratala J, et al. Contribution of a urinary antigen assay (Binax NOW) to the earlydiagnosis of pneumococcal pneumonia. Clin Infect Dis
2004;38:222-226

26. Zalacain R, Capelastegui A, Ruiz LA, et al. Streptococcus pneumoniae antigen in urine: diagnostic usefulness and impact on outcome of bacteraemic pneumococcal pneumonia in a large series of adultpatients. Respirology 2014;19:936-943.

27. Kim B, Kim J, Jo YH, et al. Prognostic value of pneumococcal urinary antigen test in community-acquired pneumonia. PLoS One 2018;13:e0200620.

28. Guchev IA, Yu VL, Sinopalnikov A, Klochkov OI, Kozlov RS, Stratchounski LS. Management of nonsevere pneumonia in military trainees with the urinary antigentest for Streptococcus pneumoniae: an innovative approach to targeted therapy. Clin Infect Dis 2005;40:1608-1616.

29. Falguera M, Ruiz-Gonzalez A, Schoenenberger JA, et al. Prospective, randomised study to compare empirical treatment versustargeted treatment on the basis of the urine antigen results in hospitalized patients with community-acquired pneumonia. Thorax 2010;65:101-106.

30. Kim IS, Koh EH, Kim S, Maeng KY, Jung HJ. Investigation of positive streptococcus pneumoniae urinary antigen test results in a Korean university hospital. Korean J Clin Microbiol 2010;13:14-18.

31. del Mar Garcia-Suarez M, Cima-Cabal MD, Villaverde R, et al. Performance of a pneumolysin enzyme-linked immunosorbent assay for diagnosis of pneumococcal infections. J Clin Microbiol 2007;45:3549-3554.

32. Tleyjeh IM, Tlaygeh HM, Hejal R, Montori VM, Baddour LM. The impact ofpenicillin resistance on short-term mortality in hospitalized adults withpneumococcal pneumonia: a systematic review and meta-analysis. Clin Infect Dis 2006;42:788-797.

33. McCabe C, Kirchner C, Zhang H, Daley J, Fisman DN. Guideline-concordanttherapy and reduced mortality and length of stay in adults with community-acquired pneumonia: playing by the rules. Arch Intern Med 2009;169:1525-1531.

34. Salinas-Botran A, Martin-Rico P, Valdivia A, Pellicer A, Esparcia O. Positive urine pneumococcal antigen test and vaccination. Med Clin (Barc) 2016;146:346-347.

35. Jain S, Self WH, Wunderink RG, et al. Community-acquired pneumonia requiring hospitalization among U.S. adults. N Engl J Med 2015;373:415-427.

36. Weinberger DM, Harboe ZB, Sanders EA, et al. Association of serotype with risk of death due to pneumococcal pneumonia: ameta-analysis. Clin Infect Dis 2010;51:692-699. 